\title{
Automatic structures for subsemigroups of Baumslag-Solitar semigroups
}

Alan J. Cain

School of Mathematics $\mathcal{E}$ Statistics, University of St Andrews North Haugh, St Andrews, Fife KY16 9SS, United Kingdom ${ }^{1}$

Email: ajcain@fc.up.pt

Web page: www.fc.up.pt/pessoas/ajcain/

\begin{abstract}
This paper studies automatic structures for subsemigroups of Baumslag-Solitar semigroups (that is, semigroups presented by $\left\langle x, y \mid\left(y x^{m}, x^{n} y\right)\right\rangle$ where $\left.m, n \in \mathbb{N}\right)$. A geometric argument (a rarity in the field of automatic semigroups) is used to show that if $m>n$, all of the finitely generated subsemigroups of this semigroup are [right-] automatic. If $m<n$, all of its finitely generated subsemigroups are left-automatic. If $m=n$, there exist finitely generated subsemigroups that are not automatic. An appendix discusses the implications of these results for the theory of Malcev presentations. (A Malcev presentation is a special type of presentation for semigroups embeddable into groups.)
\end{abstract}

\section{INTRODUCTION}

Baumslag-Solitar groups, which have presentations of the form

$$
\mathrm{Gp}\left\langle x, y \mid\left(y x^{m}, x^{n} y\right)\right\rangle,
$$

where $m, n \in \mathbb{N}$, were introduced to answer certain questions on Hopficity [BS62]. They have proved to be of independent interest, as have the semigroups with the same presentation

$$
\operatorname{Sg}\left\langle x, y \mid\left(y x^{m}, x^{n} y\right)\right\rangle .
$$

Denote the Baumslag-Solitar group ( 1 ) by $G(m, n)$ and the Baumslag-Solitar semigroup (2) by $S(m, n)$. As a consequence of Adyan's Theorem [Ady6o], the semigroup $S(m, n)$ embeds into the group $G(m, n)$ for any $m, n \in \mathbb{N}$.

The present paper studies automatic structures for finitely generated subsemigroups of Baumslag-Solitar semigroups and the results obtained confirm Jackson's [Jaco2] observation of the radical differences between BaumslagSolitar groups and semigroups. The concept of an automatic structure has

1 Current address: Centro de Matemática, Universidade do Porto, Rua do Campo Alegre 687, 4169-007 Porto, Portugal. 
been extended from groups [ECH ${ }^{+}$92] to semigroups [CRRTo1]. While automatic structures for groups have an elegant geometric characterization known as the 'fellow traveller' property, this characterization does not extend to automatic structures for general semigroups. Although two different geometric characterizations have been established for automatic structures for general semigroups [HTo6] and for a broad class that includes the right-cancellative semigroups [SSO4], these are neither as elegant nor as easy to use as the fellow traveller property. Thus, while geometric reasoning plays a vital role in the theory of automatic groups, the theory of automatic semigroups exhibits a paucity of such arguments, tending instead towards syntactic analyses.

However, automatic structures for group-embeddable semigroups do inherit many of the pleasant geometric properties of automatic groups (see [CRRo6a, Section 3] or [Caio5, Section 2.3]). Thus - for example - relatively basic geometric arguments have been used successfully by Robertson, Ruškuc, and the present author to prove that all finitely generated subsemigroups of virtually free groups are automatic [CRRo6a, Theorem 4]. In the present paper, rather more sophisticated geometric reasoning is deployed to prove that all finitely generated subsemigroups of the Baumslag-Solitar semigroup $S(m, n)$ are automatic when $m>n$ (Theorem 4.2).

The result that finitely generated subsemigroups of $S(m, n)$ are left-automatic when $m<n$ (Theorem 5.1) follows as a corollary. (See Definition 2.4 for the meaning of left-automatic.)

Hoffmann [Hofo1, Corollary 4.20] used syntactic reasoning to show that the Baumslag-Solitar semigroup $S(m, n)$ is automatic if $m>n$ and left-automatic if $m<n$; these results follow as special cases of Theorems 4.2 and 5.1.

However, as is shown in Section 6, the Baumslag-Solitar semigroup S( $m, m)$ contains finitely generated subsemigroups that are neither right- nor left-automatic. This is in some sense surprising - one would expect that the structure of a Baumslag-Solitar semigroup $S(m, n)$ is more complex when $m \neq n$ and simpler when $m=n$. Indeed, the Baumslag-Solitar group $G(m, n)$ is automatic if and only if $m=n\left[\mathrm{ECH}^{+} 92\right.$, Example 7.4.1].

Malcev presentations are a special type of semigroup presentation for semigroups embeddable into groups. Every automatic or left-automatic groupembeddable semigroup admits a finite Malcev presentation [CRRo6a, Theorem 2]. The results of this paper therefore have implications for the theory of Malcev presentations. An appendix in Section 7 discusses these implications and poses some questions for further research.

[This paper is based on Chapter 8 of the author's Ph.D. thesis [Caio5].]

\section{PRELIMINARIES}

\subsection{Words, prefixes, and suffixes}

Following $\left[\mathrm{ECH}^{+}\right.$92], the notation used in this paper distinguishes a word from the element of the semigroup or group it represents. Let $A$ be an alphabet representing a set of generators for a semigroup S. For any word $w \in A^{+}$, denote by $\bar{w}$ the element of $S$ represented by $w$. Similarly, if $A$ represents a set of generators for a group $\mathrm{G}$, let $\bar{w}$ be the element of $\mathrm{G}$ represented by $w \in\left(A \cup A^{-1}\right)^{*}$. In both cases, for any set of words $W, \bar{W}$ is the set of all elements represented by at least one word in $W$.

Denote the identity of $A^{*}$ - the empty word — by $\varepsilon$. Denote the length of 
$u \in A^{*}$ by $|u|$.

Let $u=u_{1} \cdots u_{n}$, where $u_{i} \in A$. For $t \in \mathbb{N} \cup\{0\}$, let

$$
u(t)= \begin{cases}\varepsilon & \text { if } t=0 \\ u_{1} \cdots u_{t} & \text { if } 0<t \leqslant n \\ u_{1} \cdots u_{n} & \text { if } n<t,\end{cases}
$$

and let

$$
u[t]= \begin{cases}u_{t+1} \cdots u_{n} & \text { if } 0 \leqslant t<n \\ \varepsilon & \text { if } n \leqslant t .\end{cases}
$$

So $u(t)$ is the prefix of $u$ up to and including the $t$-th letter; $u[t]$ is the suffix of $u$ after and not including the $t$-th letter. Observe that for all $t \in \mathbb{N} \cup\{0\}$, $u=u(t) u[t]$, and that if one formally assumes that $u_{t}=\varepsilon$ for $t>n$, then $u(t+1)=u(t) u_{t+1}$ and $u[t]=u_{t+1} u[t+1]$.

\subsection{Cayley graphs}

Definition 2.1. Let $S$ be a semigroup and $A$ an alphabet representing a set of generators for $S$. The Cayley graph $\Gamma(S, A)$ of $S$ with respect to $A$ is the directed graph with vertex set $S$ and, for each pair of vertices $s, t$, an edge from $s$ to $t$ labelled by $a \in A$ if and only if $s \bar{a}=t$.

Let $S$ be a semigroup that embeds in a group. If $S$ is not already a monoid, then a new vertex representing a two-sided identity can be adjoined to $\Gamma(S, A)$. Throughout this paper, assume that $\Gamma(S, A)$ has a vertex representing a twosided identity as a basepoint.

Given a word $u \in A^{+}$, let $\widehat{u}$ be the walk in $\Gamma(S, A)$ starting at the basepoint and labelled by $u$. The walk $\widehat{u}$ ends at the vertex $\bar{u}$.

Definition 2.2. Let $s, t$ be two vertices in $\Gamma(S, A)$. The [undirected] distance from $s$ to $t$, denoted $d(s, t)$, is defined to be the infimum of the lengths of the undirected paths from $s$ to $t$. [The assumption of the presence of a basepoint has the side-effect of ensuring at least one such undirected path exists.]

\subsection{Automatic semigroups}

This section contains the definitions and results for automatic semigroups that are required in the remainder of the paper.

Definition 2.3. Let $\$$ be a new symbol not in $A$. Let $A(2, \$)=\{(a, b): a, b \in$ $A \cup\{\$\}\}-\{(\$, \$)\}$ be a new alphabet. Define the mapping $\delta_{A}: A^{+} \times A^{+} \rightarrow$ $A(2, \$)^{+}$by

$$
\left(u_{1} \cdots u_{m}, v_{1} \cdots v_{n}\right) \mapsto \begin{cases}\left(u_{1}, v_{1}\right) \cdots\left(u_{m}, v_{n}\right) & \text { if } m=n \\ \left(u_{1}, v_{1}\right) \cdots\left(u_{n}, v_{n}\right)\left(u_{n+1}, \$\right) \cdots\left(u_{m}, \$\right) & \text { if } m>n \\ \left(u_{1}, v_{1}\right) \cdots\left(u_{m}, v_{m}\right)\left(\$, v_{m+1}\right) \cdots\left(\$, v_{n}\right) & \text { if } m<n\end{cases}
$$

where $u_{i}, v_{i} \in A$. The symbol $\$$ is usually called the padding symbol.

Definition 2.4. A right-automatic structure for $S$ is a pair $(A, L)$, where $A$ is a finite alphabet representing a set of generators for $S$ and $L \subseteq A^{+}$is a regular language with $\bar{L}=S$ and such that, for each $a \in A \cup\{\varepsilon\}$,

$$
\mathrm{L}_{\mathrm{a}}=\{(\mathrm{u}, v): u, v \in \mathrm{L}, \overline{\mathrm{ua}}=\bar{v}\} \delta_{\mathrm{A}}
$$


is a regular language over $A(2, \$)$. A right-automatic semigroup is a semigroup that admits a right-automatic structure.

A left-automatic structure for $S$ is a pair $(A, L)$, where $A$ is a finite alphabet representing a set of generators for $S$ and $L \subseteq A^{+}$is a regular language with $\overline{\mathrm{L}}=\mathrm{S}$ and such that, for each $\mathrm{a} \in A \cup\{\varepsilon\}$,

$$
{ }_{\mathrm{a}} \mathrm{L}=\{(u, v): u, v \in \mathrm{L}, \overline{\mathrm{au}}=\bar{v}\} \delta_{\mathrm{A}}
$$

is a regular language over $A(2, \$)$. A left-automatic semigroup is a semigroup that admits a left-automatic structure.

By default, 'automatic' means 'right-automatic'.

[Hoffmann $\mathcal{E}$ Thomas [HTo3] also considered the possibility of having the padding symbols $\$$ placed on the left rather than on the right. However, every cancellative semigroup that is right-automatic (respectively, left-automatic) with padding on the right is right-automatic (respectively, left-automatic) with padding on the left, and vice versa [HTo3, Remark 8.3]. As this paper is concerned only with group-embeddable semigroups, which are of course cancellative, it suffices to consider only padding on the right.]

Proposition 2.5 ([CRRTo1, Proposition 3.5]). If a semigroup $S$ is automatic, then so is $\mathrm{S}^{1}$, the semigroup formed by adjoining an identity to $\mathrm{S}$.

Proposition 2.6 ([DRR99, Theorem 1.1]). Let $M$ be a monoid with automatic structure $(A, L)$ and let $B$ represent a finite [semigroup] generating set for $M$. Then there exists an automatic structure $(\mathrm{B}, \mathrm{K})$ for $\mathrm{M}$.

TheOREM 2.7 ([CRRo6a, Theorem 3.14]). Let $S$ be a semigroup that embeds in a group. Let $(A, L)$ be a rational structure for $S$. Then $(A, L)$ is an automatic structure for $S$ if and only if there exists a constant $\lambda \in \mathbb{N}$ such that for all $a \in A \cup\{\varepsilon\}$, if $\mathrm{u}, v \in \mathrm{L}$ are such that $\overline{\mathrm{ua}}=\bar{v}$, then for all $\mathrm{t}$ the distance from $\overline{\mathrm{u}(\mathrm{t})}$ to $\overline{v(\mathrm{t})}$ is less than $\lambda$.

Proposition 2.8 ([CRRTo1, Proposition 2.3]). Let $\mathrm{U}$ and $\mathrm{V}$ be subsets of $\mathrm{A}^{+} \times \mathrm{A}^{+}$ such that $\mathrm{U} \delta_{\mathrm{A}}$ and $\mathrm{V} \delta_{\mathrm{A}}$ are regular. Then

$$
\left(\mathrm{U} \circ \mathrm{V}^{-1}\right) \delta_{\mathrm{A}}=\left\{(\mathrm{u}, v) \delta_{\mathrm{A}}:\left(\exists w \in \mathrm{A}^{+}\right)((\mathrm{u}, w) \in \mathrm{U} \wedge(v, w) \in \mathrm{V})\right\}
$$

is also a regular language over $\mathrm{A}(2, \$)$.

Definition 2.9. Let $A$ be an alphabet. For any word $w=w_{1} \cdots w_{n} \in A^{+}$, where $w_{i} \in A$, define $w^{\text {rev }}$ to be $w_{n} \cdots w_{1}$.

Let $R \subseteq A^{+} \times A^{+}$, and let $S=\operatorname{Sg}\langle A \mid \mathcal{R}\rangle$. Define $\mathcal{R}^{\text {rev }}$ by

$$
\mathcal{R}^{\text {rev }}=\left\{\left(u^{\text {rev }}, v^{\text {rev }}\right):(u, v) \in \mathcal{R}\right\} .
$$

Define $S^{\text {rev }}$ to be $\operatorname{Sg}\left\langle A \mid \mathcal{R}^{\text {rev }}\right\rangle$.

Notice that $\left(S^{\text {rev }}\right)^{\text {rev }}=S$.

LEMma 2.10 ([HTo3, Lemma 3.4]). The semigroup $\mathrm{S}$ is right-automatic if and only if the semigroup $S^{\text {rev }}$ is left-automatic. 


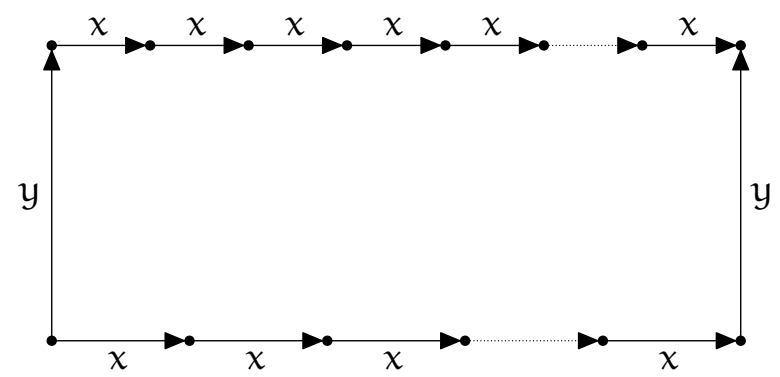

FIGURE 1. Part of the Cayley graph of the Baumslag-Solitar semigroup $S(m, n)$ : a cell describing the relation $\left(y x^{m}, x^{n} y\right)$.

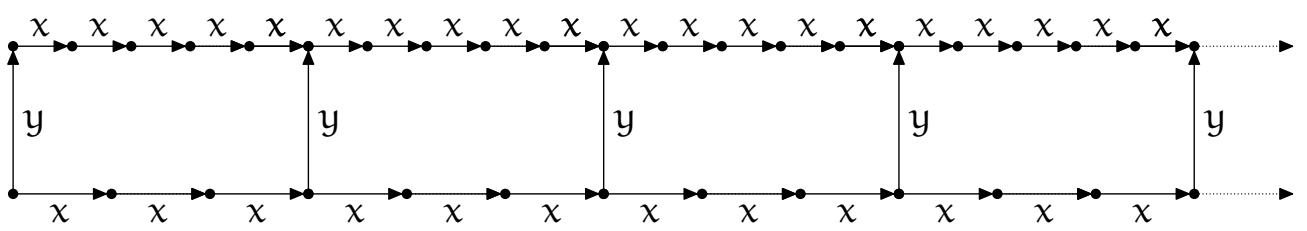

FIGURE 2. Part of the Cayley graph of the Baumslag-Solitar semigroup S(5,3).

\section{THE CAYLEY GRAPH OF THE BAUMSLAG-SOLITAR SEMIGROUPS}

Fix $m, n$ with $m>n$. The present section describes how to construct the Cayley graph of $S(m, n)$. First of all, consider a single cell describing the relation $\left(y x^{m}, x^{n} y\right)$, as shown in Figure 1. Join copies of this cell along the edges labelled $y$ as shown in Figure 2; this row of cells is infinite (to the right).

Let $\omega$ be the basepoint of the graph, from which extends an infinite horizontal row of edges each labelled by $x$. Now proceed inductively: to every infinite horizontal row $R$ of edges labelled by $x$ that has now yet been considered, add $n$ copies of the row of cells shown in Figure 2, identifying the basepoint of the $(k-1)$-th such row of cells with the $k$-th vertex from the left of R. Viewed side-on, the fragment of the graph constructed thus far is a 'fan' with $n$ spokes. [Figures 3 and 4 show this step in the construction for $m=3$ and $n=2$.] This procedure inductively constructs the Cayley graph of $S(m, n)$. Viewed side-on, this graph is an infinite $n$-ary tree, as shown in Figure 5. Select an infinite path climbing this tree and take the subset of the Cayley graph that projects to this path. Call such a subset a branch of the Cayley graph. [The construction of the Cayley graph just described is similar to that for the Baumslag-Solitar group $\mathrm{G}(\mathrm{m}, \mathrm{n})$ given by $\left[\mathrm{ECH}^{+}{ }^{92}\right.$, Section 7.4].]

A branch of the Cayley graph of $S(m, n)$ may be embedded into the Euclidean plane as shown in Figure 6. Notice that all cells describing a relation $\left(y x^{m}, x^{n} y\right)$ are similar squares, being scaled by $n / m$ as one climbs from one row to the next. Define a concept of 'horizontal distance' within rows of $x$-edges by taking the distance between vertices in the Cayley graph and extending to edges by linear interpolation. form

Each element of the Baumslag-Solitar semigroup $S(m, n)$ has a normal

$$
x^{k_{0}} y x^{k_{1}} y x^{k_{2}} \cdots y x^{k_{j}} y x^{l}
$$

where each $k_{i}$ is less than $n$; such a normal form can be obtained from any word over $x$ and $y$ by using the defining relation to move letters $x$ as far to the right as possible. Identify elements of $S(m, n)$ with these normal forms. 


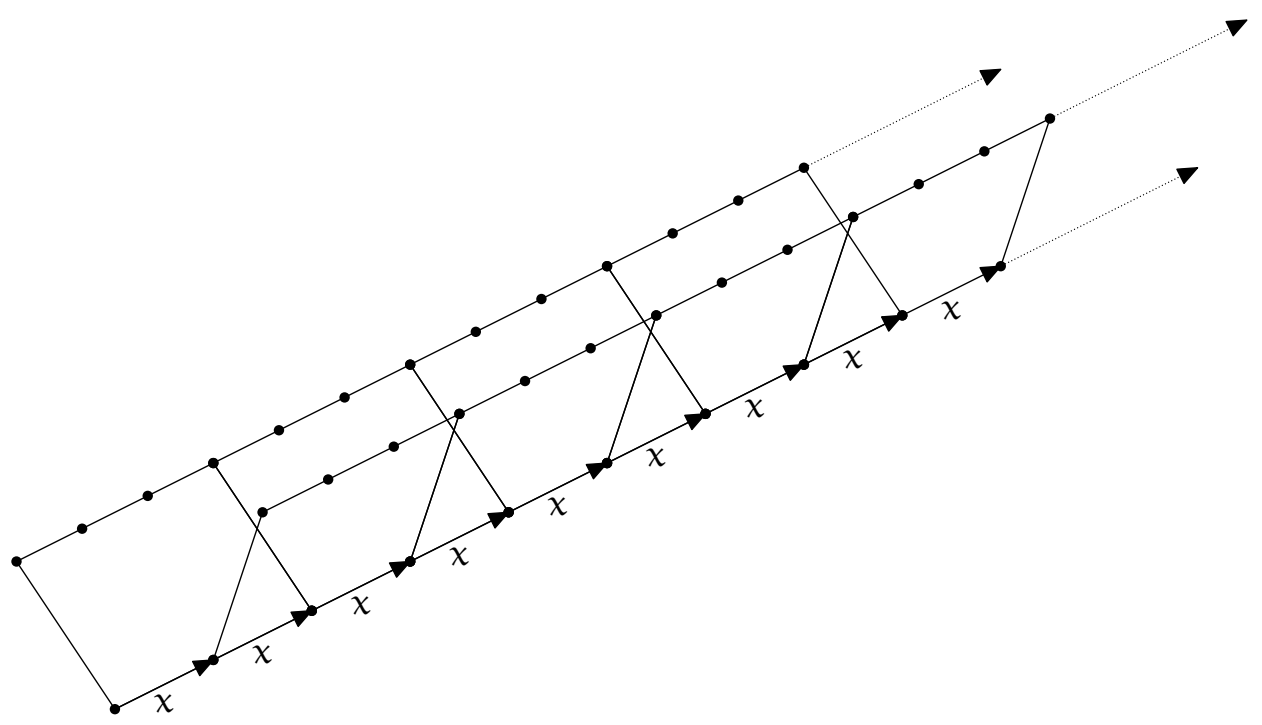

FIGURE 3. Part of the Cayley graph of the Baumslag-Solitar semigroup S(3, 2).

\section{ALL SUBSEMIGROUPS RIGHT-AUTOMATIC WHEN $m>n$}

Choose and fix $m, n \in \mathbb{N}$ with $m>n$. Let $A$ be a finite alphabet representing a subset of $S(m, n)$. Let $S$ be the subsemigroup generated by $\bar{A}$. In the Cayley graph $\Gamma(S, A)$, imagine a word $w \in A^{+}$as labelling an edge from each element $s \in S$ direct to $s \bar{w}$. In an appropriate branch of the Cayley graph of $S(m, n)$, consider an edge from $s$ to $s \bar{w}$ labelled by $w$. Embed this branch into the Euclidean plane, so that this edge becomes a straight line between $s$ and $s \bar{w}$; see Figure 7 . Now, because of the similarity of the various cells mentioned above, the angle $\theta_{w}$ between this edge and the horizontal axis is independent of $s$. Notice further that this angle must lie between 0 and $\pi / 2$.

Let $A^{\prime}$ be that subset of $A$ whose letters represent elements of $S(m, n)$ of the form $x^{k}$ for some $k \in \mathbb{N}$ (that is, letters a such that $\theta_{a}=0$ ). Let $A^{\prime \prime}=A-A^{\prime}$. For each $a \in A$; let $\beta_{a}$ be the number of symbols $y$ in $\bar{a}$. (So $a \in A^{\prime}$ if and only if $\beta_{a}=0$.) Similarly for $a \in A$, let $\gamma_{a}$ be the number of letters $x$ in $\bar{a}$. Let $g=\max \left\{\gamma_{a}: a \in A\right\}$.

Lemma 4.1. Let $X$ be a finite subset of $A^{+}-\left(A^{\prime}\right)^{+}$. Let $\mathrm{h} \in \mathbb{N}$ be arbitrary. Then there exists a horizontal distance $\alpha_{\mathrm{h}, \mathrm{x}}>\mathrm{h}$ with the following property: if edges labelled by $w, z \in X$ intersect two adjacent $x$-rows at points $\mathrm{p}, \mathrm{q}$ and $\mathrm{p}^{\prime}, \mathrm{q}^{\prime}$, respectively, and the horizontal distance between $\mathrm{p}$ and $\mathrm{q}$ exceeds $\alpha_{\mathrm{h}, \mathrm{X}}$, then the horizontal distance between $\mathrm{p}^{\prime}$ and $\mathrm{q}^{\prime}$ also exceeds $\alpha_{\mathrm{h}, \mathrm{x}}$.

[This is a purely geometrical lemma. Words $w$ and $z$ may not label lines passing through a particular choice of points $p, p^{\prime}, q$ and $q^{\prime}$.]

Proof of 4.1. Notice that if $w \in X$, then $\bar{w} \neq x^{k}$ for any $k \in \mathbb{N}$. So $\theta_{w}>0$.

Let $d$ be the horizontal distance between $p$ and $q$. Then the horizontal distance between $p^{\prime}$ and $q^{\prime}$ is given by:

$$
\frac{\mathrm{m}}{\mathrm{n}}\left|\mathrm{d}-\operatorname{cotan} \theta_{w}+\operatorname{cotan} \theta_{z}\right|,
$$

as can be seen from Figure 8. [The absolute value is needed in case the two lines cross over. The 'vertical' distance between the two x-rows is 1. Although all concepts of distance discussed here relate to the Cayley graph, one must 


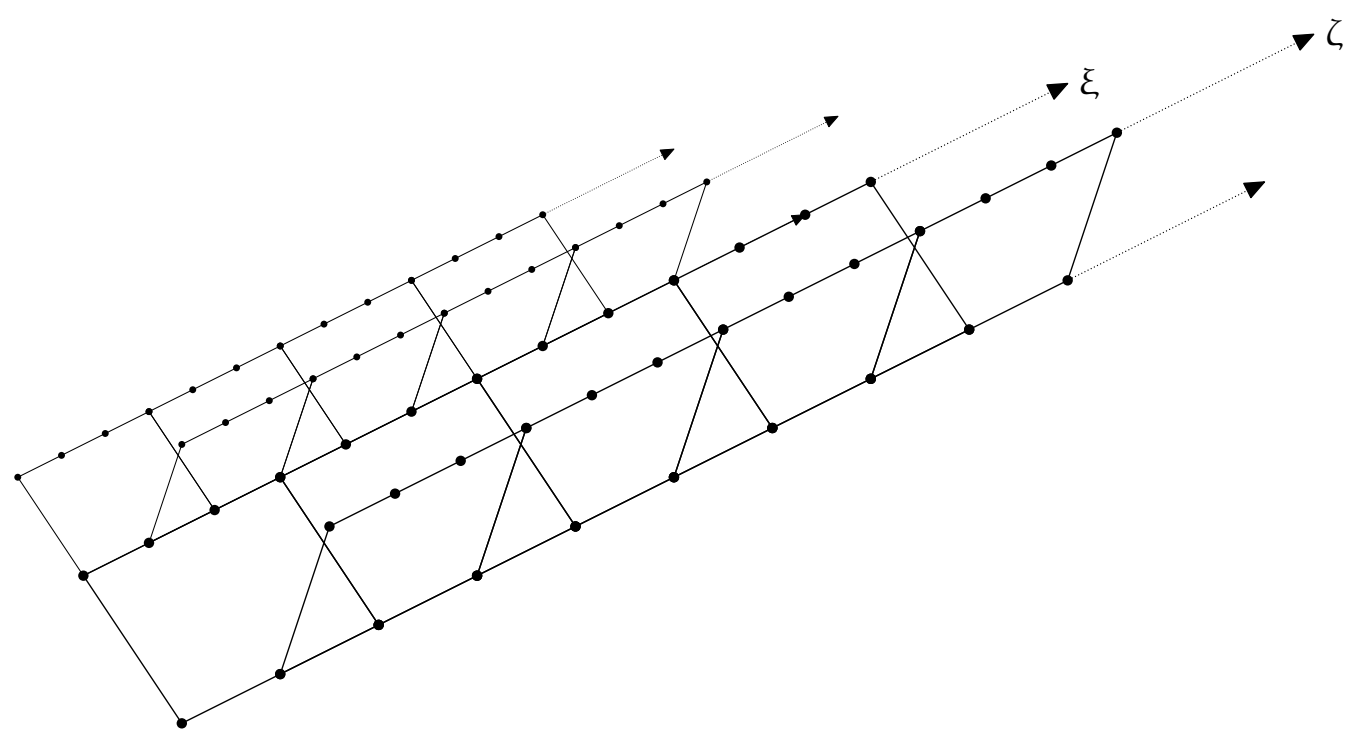

FIGURE 4. Part of the Cayley graph of the Baumslag-Solitar semigroup S(3,2). Two rows of cells have just been added to the row of x-edged marked $\xi$; the next step in the inductive construction is to add two rows of cells to the row of x-edges marked $\zeta$.

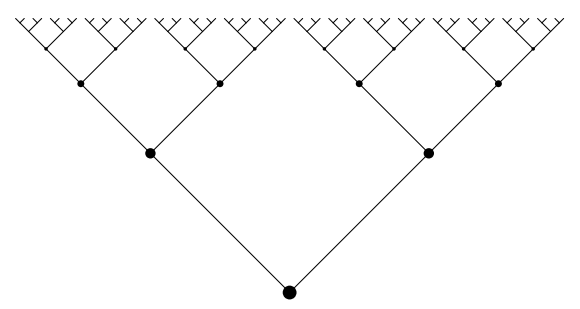

FIGURE 5. Part of the Cayley graph of the Baumslag-Solitar semigroup S(3,2) viewed 'side-on'. The only edges 'visible' are labelled by $y$; the edges labelled by $x$ are pointing into the page.

momentarily appeal to the Euclidean plane for trigonometric purposes.] Now, $m>n$ so that $m / n>1$.

Let

$$
\alpha_{h, X}=\max \left[\{h\} \cup\left\{\operatorname{cotan} \theta_{w}-\operatorname{cotan} \theta_{z}: w, z \in X\right\}\right] \frac{m}{m-n} ;
$$

since $X$ is finite the maximum exists. If $d>\alpha_{h, X}$, and $w, z \in X$, then

$$
\begin{aligned}
\mathrm{d} & >\left(\operatorname{cotan} \theta_{w}-\operatorname{cotan} \theta_{z}\right) \frac{\mathrm{m}}{\mathrm{m}-\mathrm{n}} \\
\mathrm{d}(1-\mathrm{n} / \mathrm{m}) & >\operatorname{cotan} \theta_{w}-\operatorname{cotan} \theta_{z} \\
\frac{\mathrm{m}}{\mathrm{n}}\left(\mathrm{d}-\operatorname{cotan} \theta_{w}+\operatorname{cotan} \theta_{z}\right) & >\mathrm{d} .
\end{aligned}
$$

That is, if the horizontal distance between $p$ and $q$ exceeds $\alpha_{h, X}$, then the distance between $\mathrm{p}^{\prime}$ and $\mathrm{q}^{\prime}$ is larger still. This completes the proof.

As a consequence of the defining relation $\left(y x^{m}, x^{n} y\right)$, for any $a \in A^{\prime}$,

$$
y^{k} \overline{a^{m^{k}}}=\overline{a^{n^{k}}} y^{k}
$$

and, more generally, for $b \in A^{\prime \prime}$ and $k=\beta_{b}$,

$$
\overline{\mathrm{ba}^{m^{k}}}=\overline{\mathrm{a}^{\mathrm{n}^{k}} \mathrm{~b}} \text {. }
$$




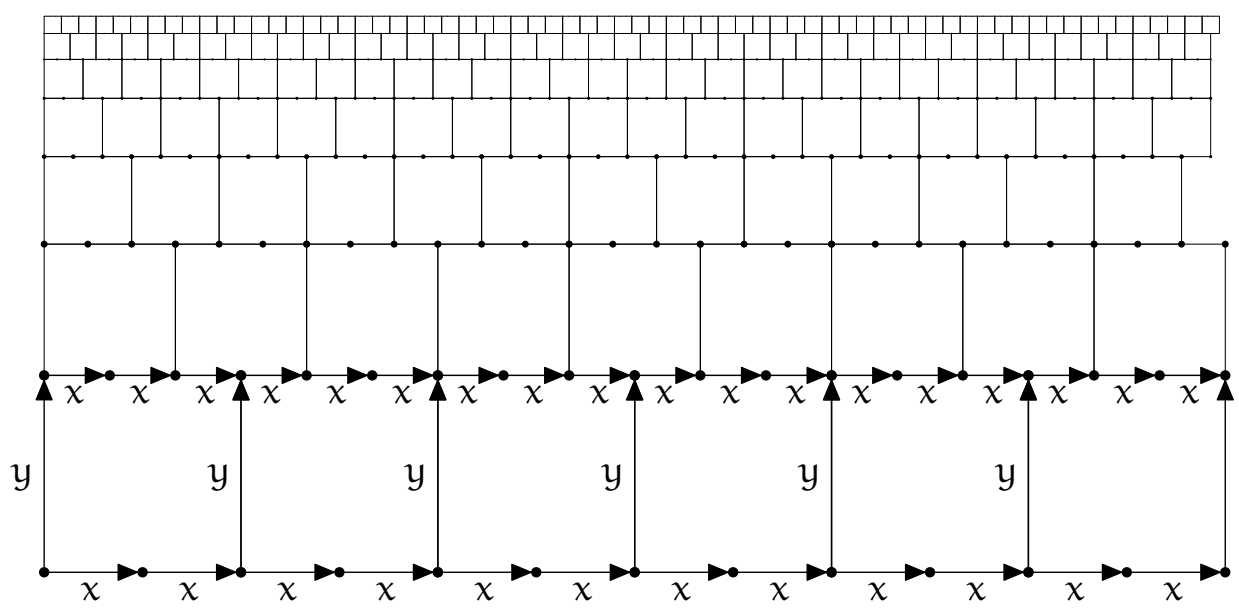

FIGURE 6. Part of a branch of the Cayley graph of the Baumslag-Solitar semigroup $\mathrm{S}(3,2)$ embedded into the Euclidean plane.

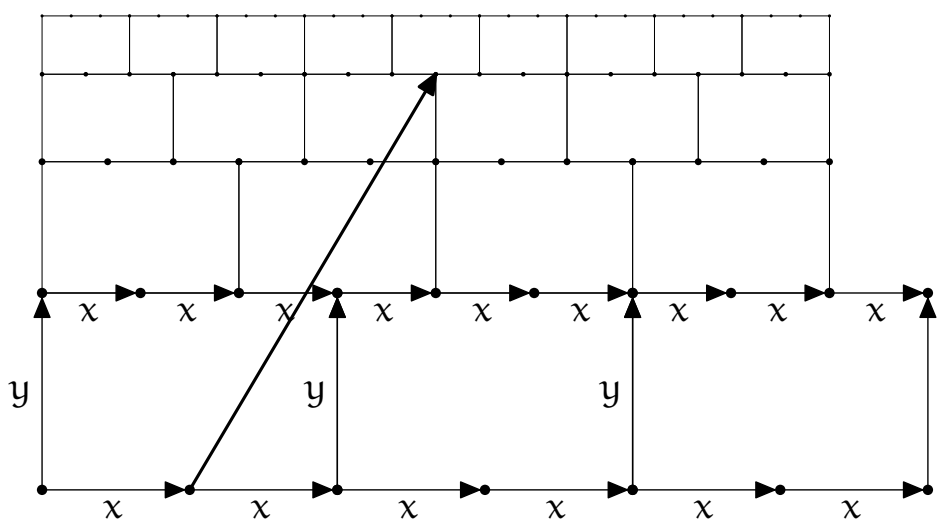

FIGURE 7. An edge running from $s$ to $s \bar{w}$, where $s=\bar{x}$ and $w=x y x y y$. Observe that the similarity of the cells in this embedding into the Euclidean plane ensures that this edge makes the same angle with each row of $x$-edges, and that this angle is independent of $s$.

Moreover, $\overline{a_{1} a_{2}}=\overline{a_{2} a_{1}}$, where $a_{1}, a_{2} \in A^{\prime}$. The upshot of this is that every element of $S$ has a representative in the set

$$
\left(A^{\prime}\right)^{*} K^{*}-\{\varepsilon\},
$$

where $\mathrm{K}$ is the finite set

$$
\left\{b a_{1}^{\alpha_{1}} \cdots a_{l}^{\alpha_{l}}: b \in A^{\prime \prime}, 0 \leqslant \alpha_{i}<m^{\beta_{b}}\right\}
$$

and $A^{\prime}=\left\{a_{1}, \ldots, a_{l}\right\}$. [In a way, this is the reverse of the set of normal forms for $S(m, n)$ - letters representing powers of $x$ are now moved as far left as possible.]

Let 1 be a new symbol representing the adjoined identity of $S^{1}$. Let $k$ be the maximum length of any element of K. Define

$$
K^{\prime}=\left\{b w 1^{\beta_{b}(k+1)-|w|-1}: b \in A^{\prime \prime}, b w \in K\right\} .
$$

The set $K^{\prime}$ consists of elements bw of $K$ padded with symbols 1 to a length that is a constant multiple of $\beta_{\mathrm{b}}$. [The large constant multiple $(k+1)$ is necessary to ensure that the string of symbols 1 always has positive length.] Define

$$
J=\left\{a 1^{\gamma_{a}-1}: a \in A^{\prime}\right\} .
$$




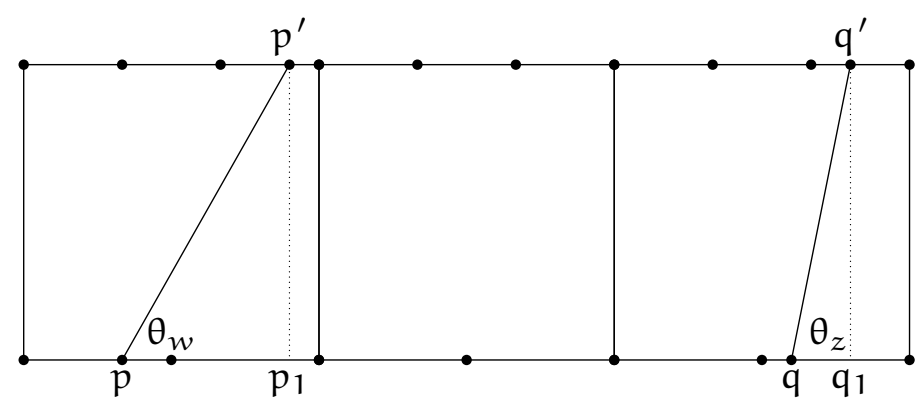

FIGURE 8. An example of the variance of horizontal distance from one $x$-row to the next. Notice that the horizontal distance from $p^{\prime}$ to $q^{\prime}$ is $m / n$ (in this case $3 / 2$ ) times that between $p_{1}$ and $q_{1}$.

(Recall that $\bar{a}=x^{\gamma}$ for each $a \in A^{\prime}$.)

Let $\mathrm{L}=\mathrm{J}^{*}\left(\mathrm{~K}^{\prime}\right)^{*}-\{\varepsilon\} \cup\{1\}$. The language $\mathrm{L}$ differs from the set (4) only by padding using symbols 1 and the addition of the word 1 to represent the adjoined identity. Therefore, since the set (4) maps onto $S$, the language $L$ maps onto $S^{1}$. The aim is now to show that $(A \cup\{1\}, L)$ is an automatic structure for $S^{1}$.

Suppose $u, v \in L$ and $a \in A \cup\{\varepsilon\}$ with $\overline{u a}=\bar{v}$. Let $u=u^{\prime} u^{\prime \prime}$ and $v=v^{\prime} v^{\prime \prime}$, where $u^{\prime}, v^{\prime} \in \mathrm{J}^{*}$ and $\mathrm{u}^{\prime \prime}, v^{\prime \prime} \in\left(\mathrm{K}^{\prime}\right)^{*}$. The paths $\widehat{u a}$ and $\widehat{v}$ run from the basepoint $\omega$ to a common vertex. As these paths never run 'downwards' through the Cayley graph, they lie in a common branch containing $\overline{u a}=\bar{v}$. Isolate such a branch and embed it into the Euclidean plane. The parts of the two paths labelled by $u^{\prime}$ and $v^{\prime}$ run along the lowest $x$-row.

Let $\mathrm{t} \in \mathbb{N} \cup\{0\}$. Suppose firstly that $\mathrm{t} \leqslant \min \left\{\left|\mathbf{u}^{\prime}\right|,\left|v^{\prime}\right|\right\}$. As any word $w$ in $\mathrm{J}$ has length equal to the number of letters $x$ in $\bar{w}$, the same holds true for any word in $\mathrm{J}^{*}$. Any prefix of a word in $\mathrm{J}^{*}$ is at $\operatorname{most} M=\max \{|w|: w \in J\}$ letters short of a member of $J^{*}$; the number of letters $x$ in $\overline{u^{\prime}(t)}$ differs by at most $M$ from $t$. Similar reasoning applies to $v^{\prime}$; the distance between $\overline{u^{\prime}(t)}$ and $\overline{v^{\prime}(t)}$ is therefore bounded.

The words $u^{\prime \prime}$ and $v^{\prime \prime}$ label subpaths from $\overline{u^{\prime}}$ and $\overline{v^{\prime}}$ to $\bar{u}$ and $\bar{v}$. It is clear that the horizontal distance from $\bar{u}$ to the intersection of path labelled by $v^{\prime \prime}$ with the $x$-row in which $\bar{u}$ lies is at most $g=\max \left\{\gamma_{a}: a \in A\right\}$. Imagine these paths as made up of 'segments' $w \in K^{\prime}$, with each $w$ labelling an edge that runs directly from $s$ to $s \bar{w}$. Consider the intersection of the paths with a given $x$-row, at points $p$ and $q$. Let the intersections with the next $x$-row be $p^{\prime}$ and $q^{\prime}$. Let $w$ and $z$ be the labels on the segments that run between $p$ and $p^{\prime}$ and $q$ and $q^{\prime}$. (These segments may of course start below $p$ and $q$ and end above $p^{\prime}$ and $q^{\prime}$.) By Lemma 4.I above, if the horizontal distance between $p$ and $q$ exceeds $\alpha_{g, K^{\prime}}$, then the distance between $p^{\prime}$ and $q^{\prime}$ also exceeds $\alpha_{g, K^{\prime}}$, regardless of $w$ and $z$. (Recall that $g$ is the maximum number of letters of $x$ in $\bar{a}$ for $a \in A$.) Therefore, since the paths labelled by $u^{\prime \prime}$ and $v^{\prime \prime}$ must eventually be a horizontal distance at most $g$ apart, the horizontal distance between their intersections with each $x$-row cannot exceed this critical value $\alpha_{g, X}$.

In particular, the points $\overline{u^{\prime}}$ and $\overline{v^{\prime}}$ can only be a bounded distance apart. Therefore $\left|u^{\prime}\right|$ and $\left|v^{\prime}\right|$ can only differ by a bounded amount.

An argument similar to that for $J^{*}$ shows that if $w$ is a prefix of a word in $\left(\mathrm{K}^{\prime}\right)^{*}$, then the length of $w$ differs from a constant multiple of the number of letters $y$ in $\bar{w}$ by only a bounded amount.

Suppose now that $t \geqslant \min \left\{\left|u^{\prime}\right|,\left|v^{\prime}\right|\right\}$. Consider the elements $\overline{u(t)}$ and $\overline{v(t)}$. 
By altering $t$ by a bounded amount, assume $v(t) \in v^{\prime}\left(K^{\prime}\right)^{*}$. By the observation in the last paragraph, the [new] elements $\overline{u(t)}$ and $\overline{v(t)}$ lie on $x$-rows that are only a bounded number of elements $y$ apart. Therefore $\overline{u(t)}$ is a bounded distance from the intersection $p$ of the subpath labelled by $u^{\prime \prime}$ with the $x$-row containing $\overline{v(t)}$. The horizontal distance between $p$ and $\overline{v(t)}$ cannot exceed $\alpha_{h, X}$. Therefore the distance between $\overline{u(t)}$ and $\overline{v(t)}$ in the Cayley graph of $\mathrm{S}(\mathrm{m}, \mathrm{n})$ is bounded. Restoring the original value for $t$ does not alter this fact.

By Theorem 2.7, $(A \cup\{1\}, L)$ is an automatic structure for $S^{1}$. The semigroup $S$ is therefore automatic by Proposition 2.5. Since $S$ was an arbitrary finitely generated subsemigroup of $S(m, n)$, this proves the following result.

THEOREM 4.2. Every finitely generated subsemigroup of the Baumslag-Solitar semigroup $\mathrm{S}(\mathrm{m}, \mathrm{n})$, where $\mathrm{m}>\mathrm{n}$, is automatic.

\section{ALL SUBSEMIGROUPS LEFT-AUTOMATIC WHEN $m<n$}

Theorem 4.2 has the following left-handed version:

THEOREM 5.1. Every finitely generated subsemigroup of the Baumslag-Solitar semigroup $\mathrm{S}(\mathrm{m}, \mathrm{n})$, where $\mathrm{m}<\mathrm{n}$, is left-automatic.

Proof of 5.1. Note first of all that

$$
S(m, n)^{\text {rev }}=S g\left\langle x, y \mid\left(y x^{m}, x^{n} y\right)\right\rangle^{\text {rev }}=S g\left\langle x, y \mid\left(x^{m} y, y x^{n}\right)\right\rangle=S(n, m) .
$$

Let $S$ be a finitely generated subsemigroup of $S(m, n)$. Then $S^{\text {rev }}$ is a subsemigroup of $S(n, m)$ and is therefore right-automatic by Theorem 4.2. So $S$ itself is left-automatic by Lemma 2.10.

\section{NON-AUTOMATIC SUBSEMIGROUPS WHEN $m=n$}

This section shows that the Baumslag-Solitar semigroup $S(m, m)$, where $m \geqslant 2$ contains finitely generated subsemigroups that are neither rightnot left-automatic. First of all, Proposition 6.I shows that $\mathrm{S}(\mathrm{m}, \mathrm{m})$ contains the direct product of a free semigroup of rank $m$ and the natural numbers. Example 6.2 then explicitly exhibits a finitely generated non-automatic subsemigroup of this direct product.

Proposition 6.1. The Baumslag-Solitar semigroup $\mathrm{S}(\mathrm{m}, \mathrm{m})$ contains the semigroup $\left\{p_{1}, \ldots, p_{m}\right\}^{*} \times(\mathbb{N} \cup\{0\})-\{(\varepsilon, 0)\}:$ the free product of the free monoid on $m$ letters and the natural numbers (including zero) with the identity $(\varepsilon, 0)$ removed.

Proof of 6.1. Let $A=\left\{p_{1}, \ldots, p_{m}, r\right\}$ be an alphabet representing elements of $\mathrm{S}(\mathrm{m}, \mathrm{m})$ as follows

$$
\overline{p_{i}}=x^{i-1} y \text { for each } i \text {, and } \bar{r}=x^{m} .
$$

Let $S$ be the subsemigroup of $S(m, m)$ generated by $\bar{A}$. The aim is to show that $S$ is presented by

$$
\left.\operatorname{Sg}\langle A|\left(p_{i} r, r p_{i}\right) \text { for all } i\right\rangle \text {. }
$$

To prove this, note firstly that every relation $\left(p_{i} r, r p_{i}\right)$ holds in $S$. Define a set of normal forms $N=\left\{p_{1}, \ldots, p_{m}\right\}^{*} r^{*}-\{\varepsilon\}$. Every element of $S$ has 
such a normal form: letters $r$ can be moved to the left of all letters $p_{i}$ using the defining relations. Consider any element of $s \in S$. Suppose first that $s$ contains some letter $y$. If $s$ begins $x^{i-1} y \cdots$, then any word in $N$ representing it must begin $p_{i} \cdots$. On the other hand, if $s$ contains no letters $y$, then $s=x^{m \alpha}$ for some $\alpha \in \mathbb{N}$ and the normal form word representing it is $r^{\alpha}$. In the first case, one can cancel the $x^{i-1} y$ and iterate this reasoning to obtain the entire normal form word representing $\mathrm{s}$. Thus $\mathrm{N}$ is a set of unique normal forms for $S$, and so $S$ has the given presentation.

Thus $\mathrm{S}$ is isomorphic to $\left\{\mathrm{p}_{1}, \ldots, \mathrm{p}_{\mathrm{m}}\right\}^{*} \times(\mathbb{N} \cup\{0\})-\{(\varepsilon, 0)\}$.

ExAmPle 6.2. Let $A=\{a, b, c, d, e, f, g, h\}$ be an alphabet representing elements of $\{x, y, p, q, r\}^{*} \times(\mathbb{N} \cup\{0\})$ as follows:

$$
\begin{array}{ll}
\overline{\mathrm{a}}=\left(x^{2} p, 0\right), & \\
\overline{\mathrm{b}}=(\mathrm{qrp}, 1), & \overline{\mathrm{f}}=\left(x^{2} p q, 0\right), \\
\overline{\mathrm{c}}=(\mathrm{qr}, 0), & \overline{\mathrm{g}}=(r p q r p q, 3), \\
\overline{\mathrm{d}}=(\mathrm{pqr}, 2), & \overline{\mathrm{h}}=\left(r p y^{2}, 0\right) . \\
\overline{\mathrm{e}}=\left(p \mathrm{y}^{2}, 0\right), &
\end{array}
$$

Let $S$ be the semigroup generated by $\bar{A}$.

Notice that, since any free semigroup of finite rank embeds into the free semigroup of rank 2, which in turn embeds into any free semigroup of rank $m \geqslant 2$, the product $\{x, y, p, q, r\}^{*} \times(\mathbb{N} \cup\{0\})$ embeds into $\left\{p_{1}, \ldots, p_{m}\right\}^{*} \times(\mathbb{N} \cup\{0\})$. So this semigroup $S$ embeds into any Baumslag-Solitar semigroup $S(m, m)$ with $m \geqslant 2$ by Proposition 6.1.

Proposition 6.3. The semigroup $S$ is neither right-automatic nor left-automatic.

Proof of 6.3. First, it will be shown that $S$ is not right-automatic. [The proof is similar in spirit to that of [Caio6, Proposition 3.1].] The proof that $S$ is not left-automatic is essentially parallel; this will be discussed briefly afterwards.

First of all, notice that for each $\alpha \in \mathbb{N}$,

$$
\overline{a b^{\alpha} c d^{\alpha} e}=\left(x^{2}(p q r)^{2 \alpha+1} p y^{2}, 3 \alpha\right)=\overline{f g^{\alpha} h} .
$$

Elementary reasoning shows that for each $\alpha \in \mathbb{N} \cup\{0\}$ the elements

$$
\overline{a b^{\alpha} c^{\alpha}}=\left(x^{2}(p q r)^{2 \alpha+1}, 3 \alpha\right), \quad \overline{f g^{\alpha}}=\left(x^{2}(p q r)^{2 \alpha} p q, 3 \alpha\right)
$$

have unique representives $a b^{\alpha} c d^{\alpha}$ and $f g^{\alpha}$ over the alphabet $A$. [The more complicated case is $\left(x^{2}(\mathrm{pqr})^{2 \alpha+1}, 3 \alpha\right)$ : considering the free semigroup component shows that any word representing this element must be of the form $\mathrm{ab}^{\beta} \mathrm{cd}^{\gamma}$ with $\beta+\gamma=2 \alpha$. The $\mathbb{N}$-component requires that $\beta+2 \gamma=3 \alpha$. Together this forces $\beta=\gamma=\alpha$.]

Suppose, with the aim of obtaining a contradiction, that $S$ is automatic. Then, by Proposition 2.5, so is $S^{1}$. Proposition 2.6 implies that $S^{1}$ has an automatic structure $(C, L)$, where $C=A \cup\{1\}$. (The new symbol 1 represents the adjoined identity of $S^{1}$.) Let $\phi: C^{*} \rightarrow A^{*}$ map $w \in C^{*}$ to the word over $A$ formed by deleting any symbols 1 from $w$. Obviously $\overline{w \phi}=\bar{w}$.

Proposition 2.8 shows that the language

$$
\begin{aligned}
\mathrm{L}_{e} \circ \mathrm{L}_{\mathrm{h}}^{-1} & =\left\{(\mathrm{u}, w) \delta_{\mathrm{C}}: \mathrm{u}, w \in \mathrm{L}, \overline{\mathrm{ue}}=\bar{w}\right\} \circ\left\{(w, v) \delta_{\mathrm{C}}: w, v \in \mathrm{L}, \overline{v \mathrm{~h}}=\bar{w}\right\} \\
& =\left\{(\mathrm{u}, v) \delta_{\mathrm{C}}: \mathrm{u}, v \in \mathrm{L}, \overline{\mathrm{ue}}=\overline{v \mathrm{~h}}\right\}
\end{aligned}
$$


is regular. Let $\mathrm{N}$ be the number of states in a finite state automaton $\mathcal{A}$ recognizing $\mathrm{L}_{e} \circ \mathrm{L}_{\mathrm{h}}^{-1}$.

For each $\alpha \in \mathbb{N} \cup\{0\}$, let $u_{\alpha}$ and $v_{\alpha}$ be representatives in $L$ of the elements $\overline{\mathrm{ab}^{\alpha} \mathrm{cd}^{\alpha}}$ and $\overline{\mathrm{fg}^{\alpha}}$, respectively. Since $\overline{\mathrm{ab}^{\alpha} \mathrm{cd}^{\alpha}}$ has a unique representative over $A$, it is clear that $u_{\alpha} \phi=a b^{\alpha} c d^{\alpha}$. Similarly, $v_{\alpha} \phi=f g^{\alpha}$. (So $u_{\alpha}$ and $v_{\alpha}$ are the words $a b^{\alpha} \mathrm{cd}^{\alpha}$ and $\mathrm{fg}^{\alpha}$ with some symbols 1 possibly inserted.) By its definition, the language $\mathrm{L}_{e} \circ \mathrm{L}_{h}^{-1}$ contains $\left(\mathrm{u}_{\alpha}, v_{\alpha}\right) \delta_{\mathrm{C}}$ for all $\alpha \in \mathbb{N} \cup\{0\}$.

Fix $\alpha>N$. Consider the automaton $\mathcal{A}$ reading $\left(u_{\alpha}, v_{\alpha}\right) \delta_{C}$, and the states it enters immediately after reading each of the letters $b$ from the word $u_{\alpha}$. Since the number of letters $b$ exceeds $N$, the automaton enters the same state after reading two different letters $b$. Let $u^{\prime}$ and $u^{\prime} u^{\prime \prime}$ be the prefixes of $u_{\alpha}$ up to and including these two different letters $b$. That is, $u^{\prime} \phi=a b^{\beta},\left(u^{\prime} u^{\prime \prime}\right) \phi=a b^{\gamma}$, for some $\beta, \gamma \in \mathbb{N}$ with $\gamma>\beta$. Let $v^{\prime}$ and $v^{\prime} v^{\prime \prime}$ be prefixes of $v_{\alpha}$ of the same lengths as $u^{\prime}$ and $u^{\prime} u^{\prime \prime}$, respectively. The subword $v^{\prime \prime}$ is such that $v^{\prime \prime} \phi=f g^{\eta}$ or $v^{\prime \prime} \phi=g^{\eta}$ for some $\eta \in \mathbb{N} \cup\{0\}$. (The former possibility arises because $v^{\prime}$ may be a string of symbols 1.) So pumping $\left(u^{\prime \prime}, v^{\prime \prime}\right) \delta_{C}$ shows that there is $(w, z) \delta_{C} \in \mathrm{L}_{e} \circ \mathrm{L}_{h}^{-1}$ with either

$$
w \phi=a b^{\beta} b^{2(\gamma-\beta)} b^{\alpha-\gamma} c d^{\alpha} \text { and } z \phi=f g^{\eta} f g^{\alpha},
$$

or

$$
w \phi=\mathrm{ab}^{\beta} \mathrm{b}^{2(\gamma-\beta)} \mathrm{b}^{\alpha-\gamma} \mathrm{cd}^{\alpha} \text { and } z \phi=\mathrm{fg}^{\alpha+\eta} .
$$

In both cases, by the definition of $\mathrm{L}_{e} \circ \mathrm{L}_{\mathrm{h}}^{-1}$, it follows that $\overline{w e}=\overline{z h}$. In the former case, this implies that

$$
\left(x^{2}(p q r)^{2 \alpha+\gamma-\beta+1} p y^{2}, 3 \alpha+\gamma-\beta\right)=\left(x^{2}(p q r)^{2 \eta} p q x^{2}(p q r)^{2 \alpha+1} p y^{2}, 3 \alpha+3 \eta\right) ;
$$

in the latter, that

$$
\left(x^{2}(\mathrm{pqr})^{2 \alpha+\gamma-\beta+1} \mathrm{py}^{2}, 3 \alpha+\gamma-\beta\right)=\left(x^{2}(\mathrm{pqr})^{2 \alpha+2 \eta+1} \mathrm{py}^{2}, 3 \alpha+3 \eta\right) .
$$

In the former case, the free semigroup components do not match, which is a contradiction. In the latter, for the free semigroup components to match, $2 \eta=\gamma-\beta$. But for the $\mathbb{N}$-components to match, $3 \eta=\gamma-\beta$, which forces $\gamma-\beta=0$, contradicting $\gamma>\beta$. So both cases lead to a contradiction: hence $S$ cannot be automatic.

To see that $S$ is not left-automatic, proceed in the same way, but use elements $\overline{b^{\alpha} c^{\alpha} e}$ and $\overline{g^{\alpha} h}$, and the regular language ${ }_{a} L{ }_{f} L^{-1}$.

\section{APPENDIX: MALCEV PRESENTATIONS}

Malcev presentations are a special type of semigroup presentation for semigroups embeddable into groups. Informally, a Malcev presentation defines a semigroup by means of generators, defining relations, and a rule of group-embedability. This rule of group-embeddability is worth an infinite number of defining relations, in the sense that a semigroup can admit a finite Malcev presentation but no finite ordinary presentation. The present section discusses what implications Theorems 4.2 and 5.1 and Example 6.2 have, in the light of Theorem 7.3 below, for the theory of Malcev presentations.

Malcev presentations were introduced by Spehner in 1977 [Spe77], though they are based on Malcev's necessary and sufficient condition for the embeddability of a semigroup in a group [Mal39] (see also [CP67, Chapter 12]). The 
theory of Malcev presentations was relatively inactive until recent work by the present author and collaborators [Caio5, Caiog, CRRo6a, CRRo6b]; see also the survey article [Caio7].

Definition 7.1. Let $S$ be any semigroup. A congruence $\sigma$ on $S$ is a Malcev congruence if $S / \sigma$ is embeddable in a group.

If $\left\{\sigma_{i}: i \in I\right\}$ is a set of Malcev congruences on $S$, then $\sigma=\bigcap_{i \in I} \sigma_{i}$ is also a Malcev congruence on $S$. This is true because $S / \sigma_{i}$ embeds in a group $G_{i}$ for each $i \in I$, so $S / \sigma$ embeds in $\prod_{i \in I} S / \sigma_{i}$, which in turn embeds in $\prod_{i \in I} G_{i}$. The following definition therefore makes sense.

Definition 7.2. Let $A^{+}$be a free semigroup; let $\rho \subseteq A^{+} \times A^{+}$be any binary relation on $A^{+}$. Denote by $\rho^{\mathrm{M}}$ the smallest Malcev congruence containing $\rho$ - namely,

$$
\rho^{\mathrm{M}}=\bigcap\left\{\sigma: \sigma \supseteq \rho, \sigma \text { is a Malcev congruence on } A^{+}\right\} .
$$

Then $\operatorname{SgM}\langle A \mid \rho\rangle$ is a Malcev presentation for [any semigroup isomorphic to] $A^{+} / \rho^{M}$. If both $A$ and $\rho$ are finite, the the Malcev presentation $\operatorname{SgM}\langle A \mid \rho\rangle$ is said to be finite.

A group-embeddable semigroup is Malcev coherent if all of its finitely generated subsemigroups admit finite Malcev presentations.

Several classes of Malcev coherent semigroups are known: virtually free groups [CRRo6b, Theorem 3], virtually nilpotent groups [CRRo6a, Theorem 1], direct products of virtually free and abelian groups [Caiog, Theorem 2], and free products of free monoids and abelian groups [CRRo6a, Theorem 6]. [Each of these classes contain finitely generated semigroups that do not admit finite ordinary presentations; for this reason, their Malcev coherence is of interest. See [Caio7, Table 3] for a list of semigroups known to be Malcev coherent or not Malcev coherent.]

THEOREM 7.3 ([CRRo6a, Theorem 2]). Every right- or left-automatic group-embeddable semigroup admits a finite Malcev presentation.

(Although [CRRo6a] only contains the proof for right-automatic semigroups, the proof for left-automatic semigroups is almost identical.)

Since every finitely generated subsemigroup of a Baumslag-Solitar semigroup $S(m, n)$ with $m \neq n$ is either right- or left-automatic (Theorems 4.2 and 5.1), and since every right- or left-automatic group-embeddable semigroup admits a finite Malcev presentation (Theorem 7.3), the following result obtains:

Corollary 7.4. The Baumslag-Solitar semigroup $\mathrm{S}(\mathrm{m}, \mathrm{n})$, where $\mathrm{m} \neq \mathrm{n}$, is Malcev coherent.

This raises the following question:

Open Problem 7.5. Are the Baumslag-Solitar semigroups $S(m, m)$ (where $m \geqslant 2$ ) Malcev coherent?

[Notice that $S(1,1)$ is abelian and so Malcev coherent by Rédei's theorem [Réd65].] The author conjectures that Open Problem 7.5 has a positive answer. However, the conclusions of Section 6 rule out a proof using automatic structures.

The following question naturally arises from the coherence of BaumslagSolitar groups [Krogo]: 
Open Problem 7.6. Are Baumslag-Solitar groups Malcev coherent?

Baumslag [Bau74, Section B] asks whether all one-relator groups are coherent. Some progress has been made on this front [KS70, MW05]. It is therefore natural, although perhaps precipitate, to pose the following question:

Open Problem 7.7. Are all one-relator groups Malcev coherent?

A restricted version of this question that may be easier to answer is the following:

Open Problem 7.8. Are all one-relation cancellative semigroups Malcev coherent? (Adyan's Theorem [Ady6o] ensures that one-relation cancellative semigroups are group-embeddable.)

\section{REFERENCES}

[Ady6o] S. I. Adyan. 'On the embeddability of semigroups in groups'. Soviet Math. Dokl., I (1960), pp. 819-821. [Translated from the Russian.].

[Bau74] G. Baumslag. 'Some problems on one-relator groups'. In Proceedings of the Second International Conference on the Theory of Groups (Australian Nat. Univ., Canberra, 1973), pp. 75-81. Lecture Notes in Math., Vol. 372, Berlin, 1974. Springer. DoI: 10.1007/BFboo65160.

[BS62] G. Baumslag $\mathcal{E}$ D. Solitar. 'Some two-generator one-relator nonHopfian groups'. Bull. Amer. Math. Soc., 68 (1962), pp. 199-201. DoI: 10.1090/Sooo2-9904-1962-10745-9.

[Caio5] A. J. Cain. Presentations for Subsemigroups of Groups. Ph.D. Thesis, University of St Andrews, 2005. URL: www-groups.mcs.st-andrews.ac.uk/ alanc/pub/c_phdthesis.pdf.

[Caio6] A. J. Cain. 'A group-embeddable non-automatic semigroup whose universal group is automatic'. Glasg. Math. J., 48, no. 2 (2006), pp. 337-342. DOI: 10.1017/s0017089506003107.

[Caio7] A. J. Cain. 'Malcev presentations for subsemigroups of groups - a survey'. In C. M. Campbell, M. Quick, E. F. Robertson, \& G. C. Smith, eds, Groups St Andrews 2005 (Vol. 1), no. 339 in London Mathematical Society Lecture Note Series, pp. 256-268, Cambridge, 2007. Cambridge University Press.

[Caiog] A. J. Cain. 'Malcev presentations for subsemigroups of direct products of coherent groups'. J. Pure Appl. Algebra, 213, no. 6 (2009), pp. 977-990. DOI: 10.1016/j.jpaa.2008.10.006.

[CP67] A. H. Clifford E G. B. Preston. The Algebraic Theory of Semigroups (Vol. II). No. 7 in Mathematical Surveys. American Mathematical Society, Providence, R.I., 1967.

[CRRo6a] A. J. Cain, E. F. Robertson, $\mathcal{E}$ N. Ruškuc. 'Subsemigroups of groups: presentations, Malcev presentations, and automatic structures'. J. Group Theory, 9, no. 3 (2006), pp. 397-426. DoI: 10.1515/jgt.2006.027.

[CRRo6b] A. J. Cain, E. F. Robertson, E N. Ruškuc. 'Subsemigroups of virtually free groups: finite Malcev presentations and testing for freeness'. Math. Proc. Cambridge Philos. Soc., 141, no. 1 (2006), pp. 57-66. DoI: 10.1017/s0305004106009236.

[CRRTo1] C. M. Campbell, E. F. Robertson, N. Ruškuc, \& R. M. Thomas. 'Automatic semigroups'. Theoret. Comput. Sci., 250, no. 1-2 (2001), pp. 365-391. DOI: 10.1016/S0304-3975(99)oo151-6. 
[DRR99] A. J. Duncan, E. F. Robertson, $\mathcal{E}$ N. Ruškuc. 'Automatic monoids and change of generators'. Math. Proc. Cambridge Philos. Soc., 127, no. 3 (1999), pp. 403-409. DoI: 10.1017/So305004199003722.

$\left[\mathrm{ECH}^{+}{ }^{92}\right]$ D. B. A. Epstein, J. W. Cannon, D. F. Holt, S. V. F. Levy, M. S. Paterson, $\mathcal{E}$ W. P. Thurston. Word Processing in Groups. Jones $\mathcal{E}$ Bartlett, Boston, Mass., 1992.

[Hofo1] M. Hoffmann. Automatic Semigroups. Ph.D. Thesis, University of Leicester, 2001.

[HTo3] M. Hoffmann $\mathcal{E}$ R. M. Thomas. 'Notions of automaticity in semigroups'. Semigroup Forum, 66, no. 3 (2003), pp. 337-367. DOI: 10.1007/s002330010161.

[HTo6] M. Hoffmann $\mathcal{E}$ R. M. Thomas. 'A geometric characterization of automatic semigroups'. Theoret. Comput. Sci., 369, no. 1-3 (2006), pp. 300313. DOI: $10.1016 /$ j.tcs.2006.09.008.

[Jac02] D. A. Jackson. 'Decision and separability problems for BaumslagSolitar semigroups'. Internat. J. Algebra Comput., 12, no. 1-2 (2002), pp. 33-49. DOI: 10.1142/So218196702000857.

[Krogo] P. H. Kropholler. 'Baumslag-Solitar groups and some other groups of cohomological dimension two'. Comment. Math. Helv., 65, no. 4 (1990), pp. 547-558. DOI: 10.1007/BF02566625.

[KS70] A. Karrass \& D. Solitar. 'The subgroups of a free product of two groups with an amalgamated subgroup'. Trans. Amer. Math. Soc., 150 (1970), pp. 227-255. DOI: $10.2307 / 1995492$.

[Mal39] A. I. Malcev. 'On the immersion of associative systems in groups'. Mat. Sbornik, 6, no. 48 (1939), pp. 331-336. [In Russian.].

[MWo5] J. P. McCammond \& D. T. Wise. 'Coherence, local quasiconvexity, and the perimeter of 2-complexes'. Geom. Funct. Anal., 15, no. 4 (2005), pp. 859-927. DOI: $10.1007 /$ sooo39-005-0525-8.

[Réd65] L. Rédei. The Theory of Finitely Generated Commutative Semigroups. Pergamon Press, Oxford, 1965. [Translated from the German. Edited by N. Reilly.].

[Spe77] J. C. Spehner. 'Présentations et présentations simplifiables d'un monoïde simplifiable'. Semigroup Forum, 14, no. 4 (1977), pp. 295-329. [In French.]. DOI: 10.1007/BF02194675.

[SSo4] P. V. Silva \& B. Steinberg. 'A geometric characterization of automatic monoids'. Q. J. Math., 55, no. 3 (2004), pp. 333-356. Dor: 10.1093/qjmath/55.3.333. 